\title{
Determination of Nanocrystal Size Distribution in Magnetic Multicore Particles Including Dipole-Dipole Interactions and Magnetic Anisotropy: a Monte Carlo Study
}

\author{
Vincent Schaller ${ }^{\mathrm{a}, *}$, Göran Wahnström ${ }^{\mathrm{b}}$, Anke Sanz-Velasco ${ }^{\mathrm{a}}$, Peter Enoksson ${ }^{\mathrm{a}}$ and \\ Christer Johansson ${ }^{\mathrm{c}, \#}$ \\ ${ }^{a}$ Dept. of Microtechnology and Nanoscience - MC2, Chalmers University of Technology, 41296 Göteborg, Sweden \\ ${ }^{b}$ Dept. of Applied Physics, Chalmers University of Technology, 41296 Göteborg, Sweden \\ ${ }^{c}$ The Imego Institute, 40014 Göteborg, Sweden \\ E-mail:*vincent.schaller@chalmers.se; ${ }^{*}$ christer.johansson@imego.com
}

\begin{abstract}
A correct estimate of the size distribution (i.e., median diameter $D$ and geometric standard deviation $\sigma$ ) of the magnetic nanocrystals (MNCs) embedded in magnetic multicore particles is a necessity in most applications relying on the magnetic response of these particles. In this paper we use a Monte Carlo method to simulate the equilibrium magnetization of two types of multicore particles: (I) MNCs fused in a random compact cluster, and (II) MNCs distributed on the surface of a large carrier sphere. The simulated magnetization data are then fitted using a common method based on a Langevin equation weighted with a size distribution function. Finally, the fitting parameters $D_{m}$ and $\sigma_{m}$ are compared to the real parameters $D_{p}$ and $\sigma_{p}$ used to generate the MNCs. Our results show that fitting magnetization data with a Langevin model that neglects magnetic anisotropy and dipole-dipole interactions leads to an erroneous estimate of the size distribution of the MNCs in multicore particles. The magnitude of the error depends on the particle morphology, number of MNCs contained in the particle and magnetic properties of the MNCs.
\end{abstract}

Keywords: Magnetic multicore nanoparticles, Magnetization, Size distribution, Monte Carlo simulation, Metropolis algorithm, Magnetic anisotropy, Dipole-dipole interactions.

PACS: 75.75.-c, 75.50.Tt, 05.10.Ln

\section{INTRODUCTION}

Magnetic multicore particles containing from a few up to thousands of magnetic nanocrystals (MNCs) are increasingly used in biomedical applications, such as magnetic separation [1-2], magnetic resonance imaging (MRI) [3], magnetic hyperthermia [4-6] and biosensing [7-10]. This is due, for instance, to their higher magnetic moment compared to single-core particles.

It is known that a size distribution of the MNCs modifies the magnetic response of these particles, which results, for instance, in a degradation of the heating rate in hyperthermia [11] or a reduced sensitivity in magnetic particle imaging (MPI) [12]. Although this polydispersity is in most cases prejudicial in terms of performances, it remains a difficult task to produce uniform multicore particles with monodisperse MNCs.

Therefore, a correct estimate of the size distribution parameters (i.e., median diameter $D$ and geometric standard deviation $\sigma$ ) of the MNCs embedded in the particle is a necessity in most applications. These parameters are typically calculated by fitting the magnetization data with a Langevin function weighted by a certain size distribution function [13].

However, many studies have shown that the apparent magnetic size $D_{\mathrm{m}}$ obtained from this fitting procedure is almost systematically smaller than the real physical size $D_{\mathrm{p}}$ of the MNCs (typically estimated from TEM images) [14]. This difference is in some cases attributed only to the presence of a layer with reduced magnetization (socalled magnetic dead layer) around the MNCs, whereas intrinsic factors such as magnetic anisotropy and dipolar 
interactions are not considered. Since previous research has demonstrated that these two factors are seldom negligible in case of single-core particles [15-20], this approximation may presumably be inadequate in case of multicore particles in which the MNCs are densely packed and the effect of dipole-dipole interactions cannot be reduced for instance by dilution. It would thus be of interest to evaluate the error on the estimated parameters $D$ and $\sigma$ when using the common fitting method based on the Langevin equation which assumes noninteracting MNCs with no magnetic anisotropy.

This paper reports on the role of magnetic anisotropy and dipole-dipole interactions between MNCs on the deviation between the real and apparent size parameters in case of multicore particles. We use a Monte Carlo method to compute the magnetization curve of two different types of commonly synthesized multicore particles. Unlike experimental techniques, such computer simulations enable to easily study the specific effect of each factor independently. The simulated data are then fitted with the Langevin equation to obtain the apparent parameters $D_{m}$ and $\sigma_{m}$ which are compared to the real parameters $D_{p}$ and $\sigma_{p}$ used to generate the simulated clusters of MNCs representing the multicore particles.

\section{METHOD}

The first step of the simulation is to generate a three-dimensional (3D) cluster of $N$ spherical MNCs where the diameters are randomly chosen from a log-normal distribution function with median diameter $D_{p}$ and geometric standard deviation $\sigma_{p}$ :

$$
f_{L N}\left(D, D_{p}, \sigma_{p}\right)=\frac{1}{D \ln \sigma_{p} \sqrt{2 \pi}} \exp \left(-\frac{\ln ^{2}\left(D / D_{p}\right)}{2 \ln ^{2} \sigma_{p}}\right) .
$$

Such a distribution function is known to represent well dispersions of magnetite nanocrystals [21].

The results presented hereafter focus on two typical morphologies of magnetic multicore particles: (type I) a random cluster of densely packed MNCs, such as the commercially available nanomag ${ }^{\circledR}$ nanoparticles (Micromod $\mathrm{GmbH}$ ) and fluidMAG-D ${ }^{\circledR}$ nanoparticles (Chemicell $\mathrm{GmbH}$ ), and (type II) an ensemble of MNCs deposited on the surface of a larger carrier sphere [22]. In the first case, the cluster is generated using a procedure reported previously [23]. In brief, the first MNC is placed at the origin of the coordinate system and the subsequent MNCs are added to the contact of a randomly chosen MNC already present in the cluster and with random direction in $3 \mathrm{D}$, excluding positions resulting in an overlap between MNCs. A similar approach is used for the second particle configuration, where the MNCs are randomly added on the surface of a carrier sphere forming a monolayer where each MNC is in contact with the carrier sphere. It should be noted that the size of the carrier sphere is chosen to accommodate on its surface exactly $N$ polydisperse MNCs in a random and dense configuration. As a result, the packing density of the MNCs on the surface of the sphere and hence the average distance between nearest neighbors, can be assumed approximately independent of $N$ for a given set of parameters $D_{p}$ and $\sigma_{p}$. We observe that our method produces clusters that have a realistic microstructure (Fig. 1) when comparing with TEM and SEM images of real particle systems $[22,24]$.

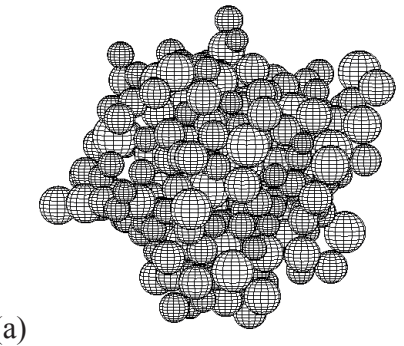

(b)

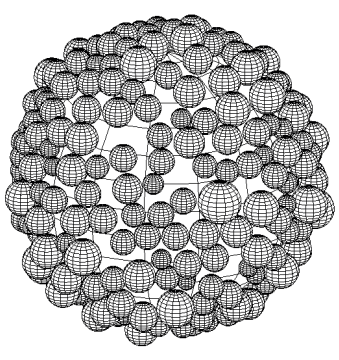

FIGURE 1. Simulated 3D clusters of spherical MNCs with log-normal size distribution (median diameter $D=10 \mathrm{~nm}$ and geometric standard deviation $\sigma=1.20$ ): (a) type I: cluster of randomly packed MNCs; (b) type II: MNCs randomly distributed on the surface of a $100 \mathrm{~nm}$ carrier sphere. 
The second step is to simulate the static magnetization curve of these clusters using a Monte Carlo method based on the Metropolis algorithm [25-26]. The magnetic dipole moment of the $i$-th single-domain nanocrystal is represented by a single vector $\boldsymbol{\mu}_{i}$ with a constant magnitude $\left\|\boldsymbol{\mu}_{i}\right\|=M_{s} V_{i}$, where $M_{s}=350 \mathrm{kA} / \mathrm{m}$ is a typical value of the intrinsic saturation magnetization for small magnetite $\left(\mathrm{Fe}_{3} \mathrm{O}_{4}\right)$ nanocrystals at room temperature, and $V_{i}=(\pi / 6) D_{i}^{3}$ is the volume of the $i$-th MNC. In addition, a unit vector $\mathbf{e}_{i}$ is assigned along the randomly oriented easy axis of each MNC.

The total energy of the $i$-th MNC is then given by:

$$
E_{i}=-\boldsymbol{\mu}_{i} \cdot \mathbf{B}-K V_{i}\left(\frac{\boldsymbol{\mu}_{i}}{\left|\boldsymbol{\mu}_{i}\right|} \cdot \mathbf{e}_{i}\right)^{2}-\frac{\mu_{0}}{4 \pi} \sum_{j \neq i}\left[\frac{3\left(\boldsymbol{\mu}_{i} \cdot \mathbf{r}_{i j}\right)\left(\boldsymbol{\mu}_{j} \cdot \mathbf{r}_{i j}\right)}{r_{i j}^{5}}-\frac{\left(\boldsymbol{\mu}_{i} \cdot \boldsymbol{\mu}_{j}\right)}{r_{i j}^{3}}\right]
$$

where the first, second and third terms on the right-hand side of Eq. (2) represent respectively: (i) the interaction energy between the dipole moment $\mu_{i}$ and the applied magnetic field $\mathbf{B}$, (ii) the uniaxial magnetic anisotropy energy with anisotropy constant $K$, and (iii) the magnetic dipole-dipole interaction energy between $\mu_{i}$ and its neighbors $\mu_{j}$. The vacuum permeability is given by $\mu_{0}=4 \pi \times 10^{7} \mathrm{~N} / \mathrm{A}^{2}$ and $\mathbf{r}_{i j}=\mathbf{r}_{i}-\mathbf{r}_{j}$ is a distance vector joining dipoles $i$ and $j$ with Euclidian norm $r_{i j}=\left\|\mathbf{r}_{i j}\right\|$. Owing to the small and finite size of the system, all neighbor pairs are considered in the summation of Eq. (2).

At each Monte Carlo iteration step, an attempt is made to change the orientation of the $N$ magnetic dipole vectors in the cluster. For each of them successively, a new direction of $\mu_{i}$ is randomly chosen with a uniform distribution in all directions. The move is accepted with probability $p=\min \left[1, \exp \left(-\Delta E_{i} / k_{B} T\right)\right]$, where $k_{B} T$ is the thermal energy and $\Delta E_{i}$ is the energy difference between the new and previous orientation of the magnetic moment $\mu_{i}$ calculated from Eq. (2). After a change in direction has been attempted for each dipole vector, the total magnetic moment of the cluster is given by the vector sum of the magnetic moments of all the MNCs:

$$
\boldsymbol{\mu}_{t o t}=\sum_{i=1}^{N} \boldsymbol{\mu}_{i}
$$

Experimental magnetization data are usually obtained from static magnetometry techniques performed either with dried or frozen samples (i.e., immobilized particles) or with particles in suspension in a liquid. Similarly, the simulation method presented here can easily be applied for both cases, either by allowing only the magnetic moments $\mu_{i}$ to rotate at each Monte Carlo step or by also rotating the entire cluster during the simulation, respectively.

In this work we focus on multicore particles in suspension and hence an additional calculation, described in details elsewhere [23], is implemented to take into account the rotation of the particle in the liquid. In brief, the Metropolis criterion is used once more at each Monte Carlo step to allow or not the rotation of the cluster based on the interaction energy between $\mu_{t o t}$ and $\mathbf{B}$, and the thermal energy $k_{B} T$.

The initial 5000 Monte Carlo steps were performed to let the system reach thermal equilibrium. Then the data were collected and averaged during the following $n=15000$ steps. All the simulations were performed at $\mathrm{T}=293 \mathrm{~K}$.

Then, the equilibrium magnetization of the cluster is defined as the projection of $\mu_{t o t}$ in the direction of the applied magnetic field, normalized by the total volume of magnetic material in the particle:

$$
M_{\text {cluster }}=\frac{1}{\sum_{i=1}^{N} V_{i}}\left\langle\boldsymbol{\mu}_{t o t} \cdot \frac{\mathbf{B}}{|\mathbf{B}|}\right\rangle
$$

where \langle\rangle denotes the average value calculated over the $n$ Monte Carlo steps after thermal equilibrium is reached.

The magnetization is simulated for 100 values of the applied magnetic field between 0 and $500 \mathrm{mT}$. These points were logarithmically spaced in order to sample more efficiently the low field region where the rate of change of the magnetization curve is higher than in the high field region. The simulation is repeated 16 times and averaged at every field with a new cluster created each time: 


$$
M=\frac{1}{16} \sum_{k=1}^{16} M_{k-\text { th cluster }}
$$

Finally, the size distribution parameters $D_{\mathrm{m}}$ and $\sigma_{\mathrm{m}}$ are evaluated by numerically fitting (in least-squares sense) the simulated magnetization data $M$ with a Langevin equation weighted by a log-normal distribution function with parameters $D_{\mathrm{m}}$ and $\sigma_{\mathrm{m}}[13]$ :

$$
M_{\text {fit }}=\frac{1}{V_{\text {tot }}} \int_{D_{\min }}^{D_{\max }} M_{S} V L\left(\frac{M_{S} V B}{k_{B} T}\right) f_{L N}\left(D, D_{m}, \sigma_{m}\right) d D
$$

The Langevin function $L$ is given by:

$$
L(\alpha)=\operatorname{coth}(\alpha)-\frac{1}{\alpha}
$$

where the Langevin factor $\alpha$ depends on the intrinsic magnetic moment of the MNCs, $M_{s} V$, the externally applied magnetic field and the temperature, i.e., $\alpha=M_{S} V B / k_{B} T$.

Since a log-normal distribution function $f_{L N}$ is used both to generate the MNCs (Eq. (1)) and to fit the simulated magnetization values (Eq. (6)), the fitting method should return estimated parameters $D_{m}$ and $\sigma_{m}$ equal to the real parameters $D_{p}$ and $\sigma_{p}$ (within a margin due to numerical accuracy) in the simple case of an ensemble of noninteracting single-domain nanocrystals with no magnetic anisotropy for which the magnetization is well described by the Langevin equation.

It is important to note here that the fitting is performed over the entire magnetization curve and not only in the low- and high-field regions using the equations derived in Ref. 13, which accuracy is subject to controversy [27].

\section{RESULTS AND DISCUSSION}

First, the consistency of the method is verified by simulating the magnetization of clusters containing $N$ noninteracting MNCs with no magnetic anisotropy, i.e., only the first term on the right-hand side of Eq. (2) is considered. In this case, the values of $D_{m}$ and $\sigma_{m}$ obtained from Eq. (6) always recover the initial parameters $D_{p}$ and $\sigma_{p}$ used to generate the MNC size distribution for any number $N$ and any particle configuration. This result is illustrated in Figs. 3-7 by the open circles coinciding with the dashed line representing $D_{m}=D_{p}$ and $\sigma_{m}=\sigma_{p}$.

Fig. 2 shows the decrease in magnetization due to the magnetic anisotropy and dipole-dipole interactions between MNCs compared to the Langevin behavior (i.e., noninteracting MNCs with no magnetic anisotropy) for a random cluster (type I) containing $N=100 \mathrm{MNCs}$. The change in the curve shape results in different parameters $D_{m}$ and $\sigma_{m}$ when fitting Eq. (6).

The effect of the uniaxial magnetic anisotropy with randomly distributed easy axes is illustrated in Fig. 3 where $D_{m}$ and $\sigma_{m}$ are plotted versus $D_{p}$ for a given $\sigma_{p}=1.20$. As seen in this figure, an uniaxial magnetic anisotropy induces a systematic under- and overestimate of the median diameter $D$ and geometric standard deviation $\sigma$, respectively. This deviation increases dramatically with the anisotropy energy, i.e., either for a larger physical median diameter $D_{p}$ or for a higher anisotropy constant $K$. Whereas the lower value of $K\left(1 \times 10^{4} \mathrm{~J} / \mathrm{m}^{3}\right)$ yields a moderate error, larger values of $K$ result in an estimated median diameter $D_{m}$ that appears to remain approximately constant (for $K=3 \times 10^{4} \mathrm{~J} / \mathrm{m}^{3}$ ) or even to decrease (for $K=5 \times 10^{4} \mathrm{~J} / \mathrm{m}^{3}$ ) for increasing values of the real median diameter $D_{p}$. On the other hand, the error on $\sigma$ increases with $D_{p}$ for a given value of $K$. These results are independent of the particle morphology. The effect of other anisotropy models such as cubic anisotropy or nonrandomly distributed easy axes may also be considered in future work. 


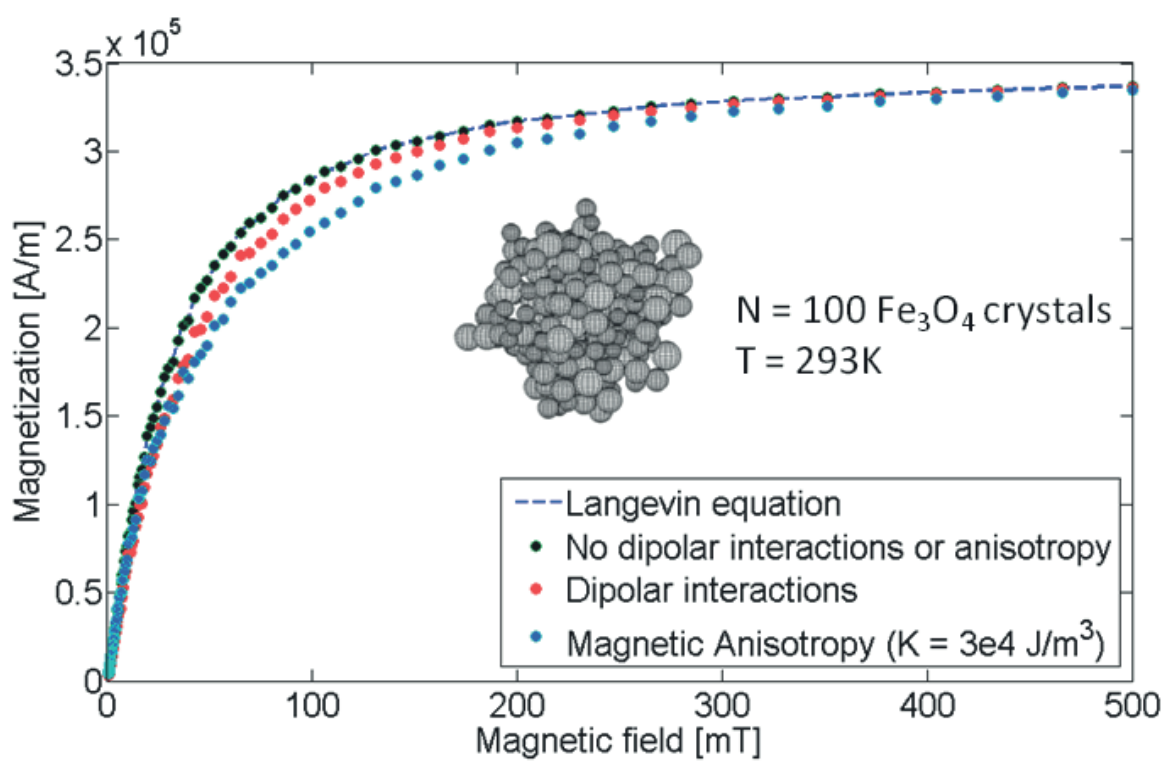

FIGURE 2. Simulated magnetization data versus applied magnetic field for a random cluster of $N=100$ MNCs with $D_{p}=10 \mathrm{~nm}$ and $\sigma_{p}=1.20$ (as illustrated in inset) at room temperature: (dashed line) analytical Langevin model; (black dots) noninteracting MNCs with no magnetic anisotropy; (red dots) dipolar-interacting MNCs with no magnetic anisotropy; (blue dots) noninteracting MNCs with magnetic anisotropy $\left(K=3 \times 10^{4} \mathrm{~J} / \mathrm{m}^{3}\right)$.

(a)

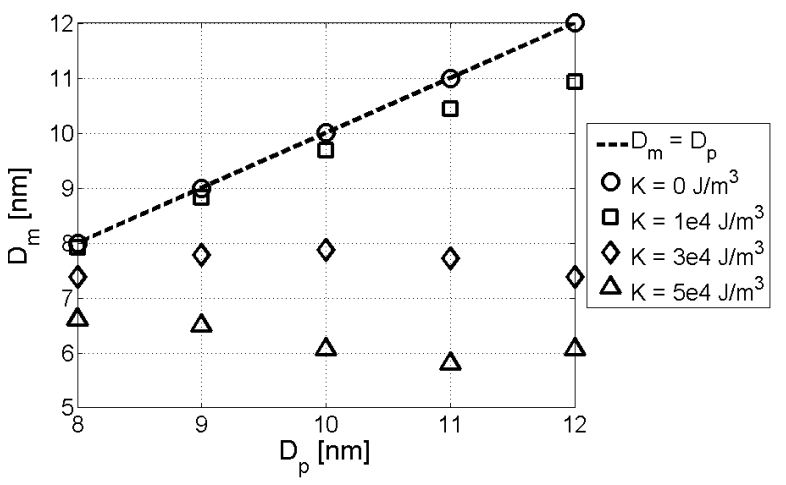

(b)

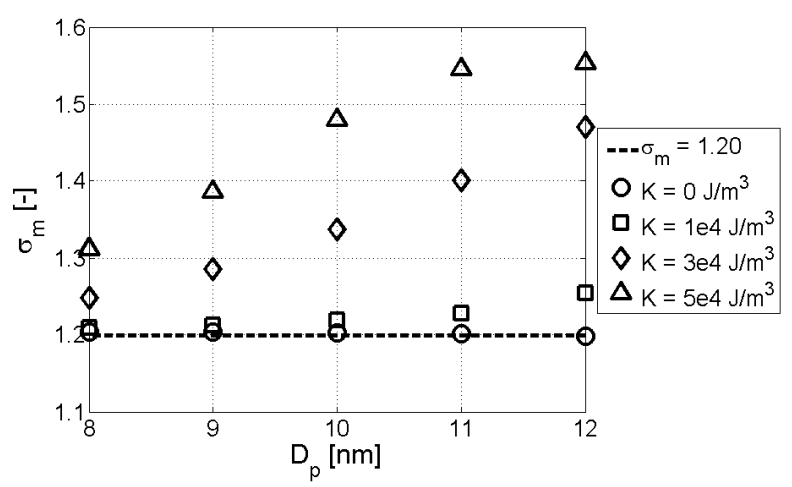

FIGURE 3. (a) $D_{m}$ versus $D_{p}$ and (b) $\sigma_{m}$ versus $D_{p}$, as a function of the anisotropy constant $K$ for $N=100$ MNCs with randomly distributed easy axes: (open circles) $K=0$, (open squares) $K=1 \times 10^{4} \mathrm{~J} / \mathrm{m}^{3}$, (open diamonds) $K=3 \times 10^{4} \mathrm{~J} / \mathrm{m}^{3}$, (open triangles)

$K=5 \times 10^{4} \mathrm{~J} / \mathrm{m}^{3}$. Dipole-dipole interactions are not included in this case. The dashed lines represent $D_{m}=D_{p}$ and $\sigma_{m}=\sigma_{p}$.

Figs. 4 and 5 show the effect of dipole-dipole interactions for $N$ magnetic nanocrystals densely packed in a randomly shaped cluster (type I) or randomly distributed on the surface of a carrier sphere (type II), respectively. Similarly to the effect of magnetic anisotropy, dipole-dipole interactions lead to a systematic underestimate of the median diameter in both particle configurations (Figs. 4 (a) and 5 (a)). On the other hand, the standard deviation may be either over- or underestimated depending on (i) the physical median diameter $D_{p}$, (ii) the particle configuration, and (iii) the size of the cluster (i.e., the number of MNCs). Interestingly, a small and large number of MNCs yield respectively an over- and underestimate of $\sigma$ for clusters of type I (Fig. 4 (b)), whereas the opposite effect is observed for clusters of type II (Fig. 5 (b)). This dependence on the cluster size can also be observed but to a less extent for $D_{m}$ where the largest deviation from $D_{p}$ occurs for the smallest clusters $(N=50)$ of type I and the largest clusters $(N=300)$ of type II.

As explained in the previous section, a larger number of MNCs in a cluster of type II requires a larger carrier sphere which in turn results in weaker dipolar interactions between MNCs located on opposite sides of the carrier 
sphere compared to densely packed MNCs as in particle of type I. This may be one of the factors contributing to the different dependence on $N$ between these two types of clusters. It would thus be of interest to study significantly larger clusters (e.g., thousands of MNCs) in future work.

(a)

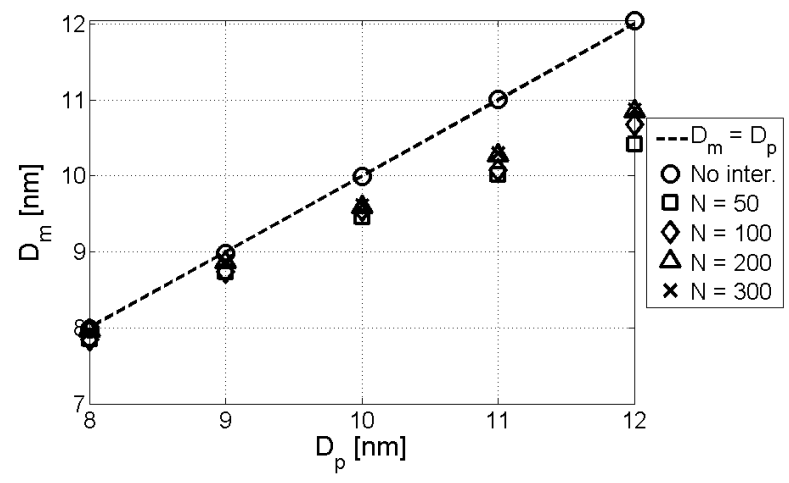

(b)

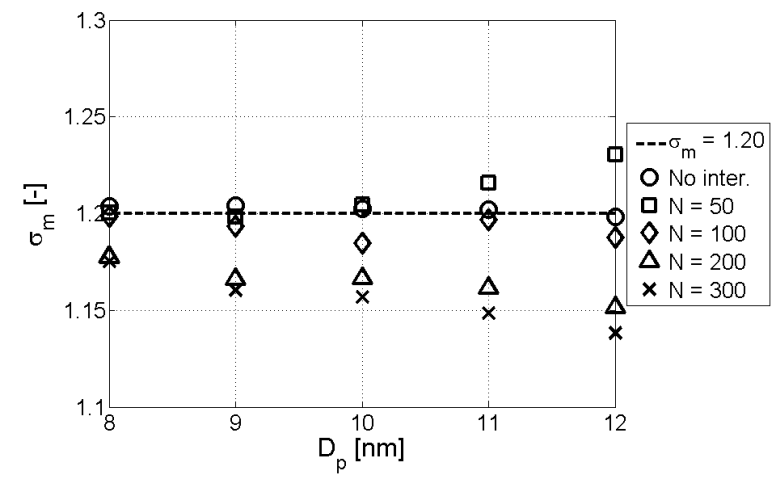

FIGURE 4. (a) $D_{m}$ versus $D_{p}$ and (b) $\sigma_{m}$ versus $D_{p}$, as a function of the number of MNCs (cluster type I) when including dipoledipole interactions: (open squares) $N=50$, (open diamonds) $N=100$, (open triangles) $N=200$, (crosses) $N=300$. The open circles show the simulated values for the non-interacting case. The magnetic anisotropy is not included in this case. The dashed lines represent $D_{m}=D_{p}$ and $\sigma_{m}=\sigma_{p}$.

(a)

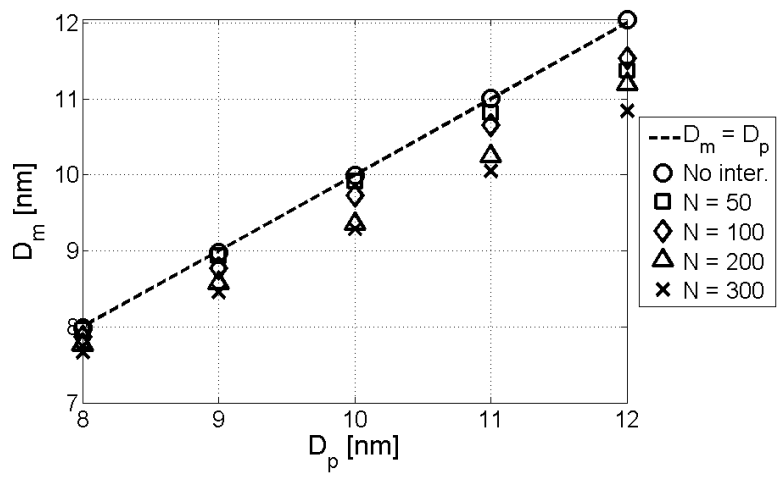

(b)

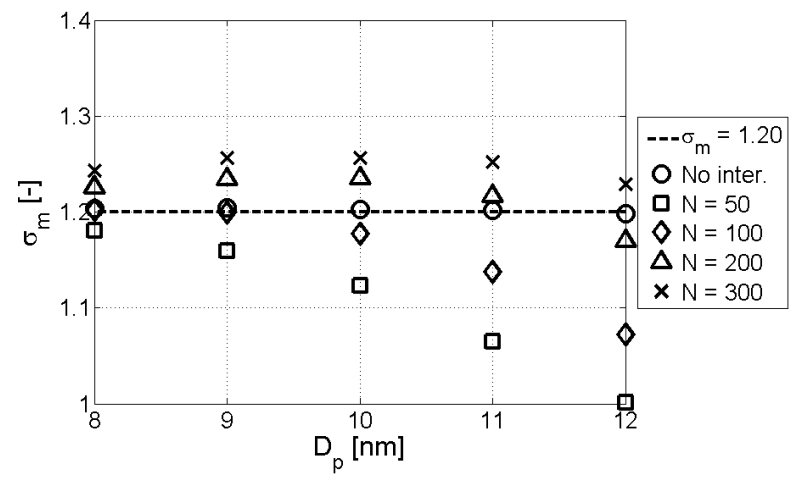

FIGURE 5. (a) $D_{m}$ versus $D_{p}$ and (b) $\sigma_{m}$ versus $D_{p}$, as a function of the number of MNCs (cluster type II) when including dipole-dipole interactions: (open squares) $N=50$, (open diamonds) $N=100$, (open triangles) $N=200$, (crosses) $N=300$. The open circles show the simulated values for the non-interacting case. The magnetic anisotropy is not included in this case. The dashed lines represent $D_{m}=D_{p}$ and $\sigma_{m}=\sigma_{p}$.

In actual particle systems, both magnetic anisotropy and dipole-dipole interactions occur simultaneously. The deviation of the fitting parameters $D_{m}$ and $\sigma_{m}$ from the real parameters $D_{p}$ and $\sigma_{p}$ when including these two intrinsic factors is shown in Figs. 6 and 7 for clusters of type I and II, respectively. The interplay between the two contributions can be seen for both $D_{m}$ and $\sigma_{m}$. The median diameter is underestimated; the error is, however, smaller than in the case of magnetic anisotropy only (Fig. 3 (a)) but significantly larger than in the case of dipole-dipole interactions only (Figs. 4 (a) and 5 (a)). The geometric standard deviation is constantly overestimated as previously observed in the case of magnetic anisotropy only (Fig. 3 (b)). It is interesting to note that the different effect of the cluster size (i.e., number of MNCs) depending on the particle configuration is similar to what has been discussed in the case of dipole-dipole interactions only. Namely, values of $\sigma_{m}$ are larger (smaller) for $N=50$ than for $N=300$ of type I (type II). These results indicate that neglecting both magnetic anisotropy and dipole-dipole interactions leads to an erroneous estimate of the size distribution that depends on the size and configuration of the cluster. 
These findings are consistent with experimental work reported by others where the physical size $D_{p}$ of the MNCs in multicore particles is almost systematically larger than the apparent magnetic size $D_{m}$ extracted from the fitting procedure to the magnetization curve.

(a)

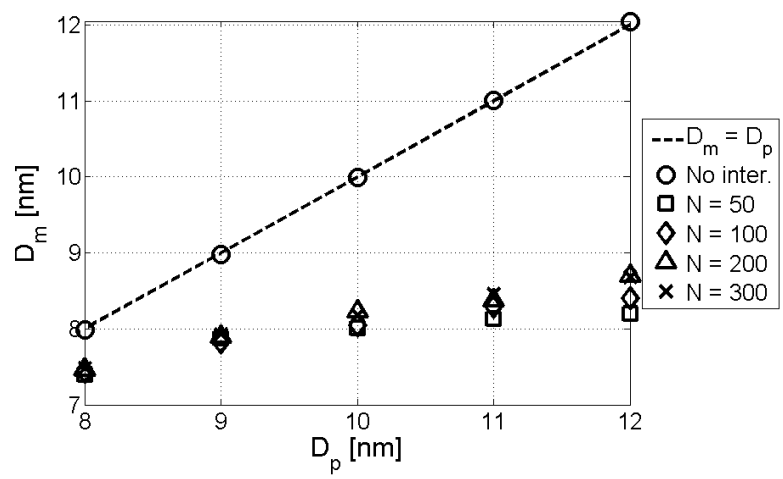

(b)

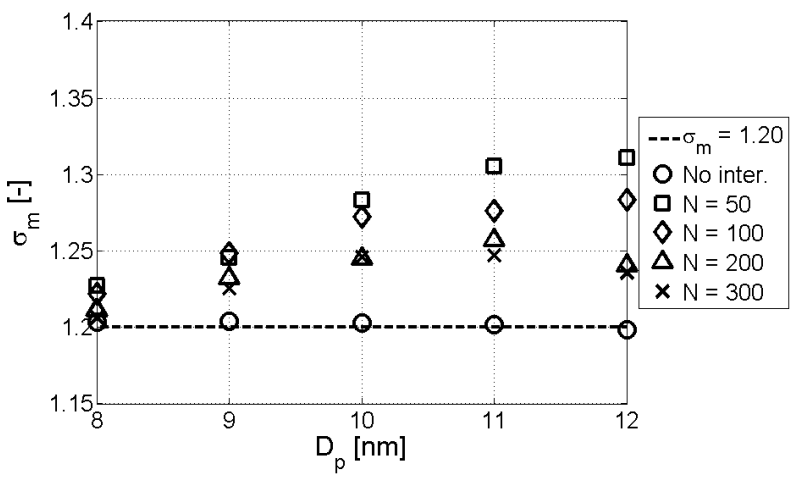

FIGURE 6. (a) $D_{m}$ versus $D_{p}$ and (b) $\sigma_{m}$ versus $D_{p}$, as a function of the number of MNCs (cluster type I) when including both the magnetic anisotropy $\left(K=3 \times 10^{4} \mathrm{~J} / \mathrm{m}^{3}\right)$ and dipole-dipole interactions: (open squares) $N=50$, (open diamonds) $N=100$, (open triangles) $N=200$, (crosses) $N=300$. The open circles show the simulated values for the noninteracting MNCs with no anisotropy. The dashed lines represent $D_{m}=D_{p}$ and $\sigma_{m}=\sigma_{p}$.

(a)

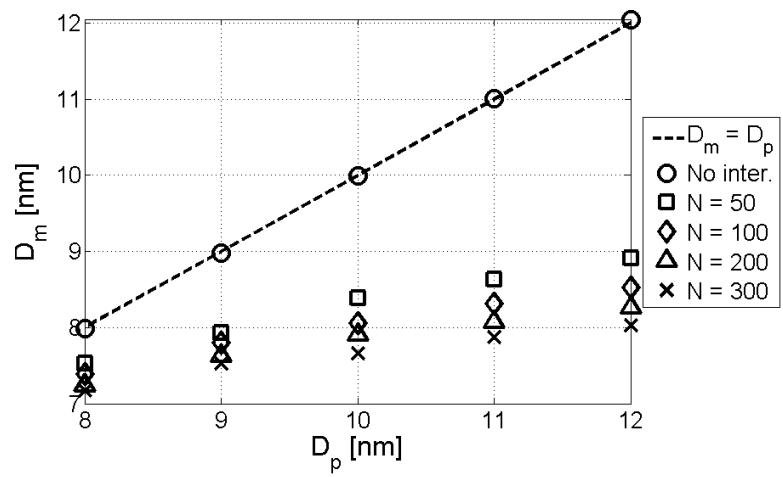

(b)

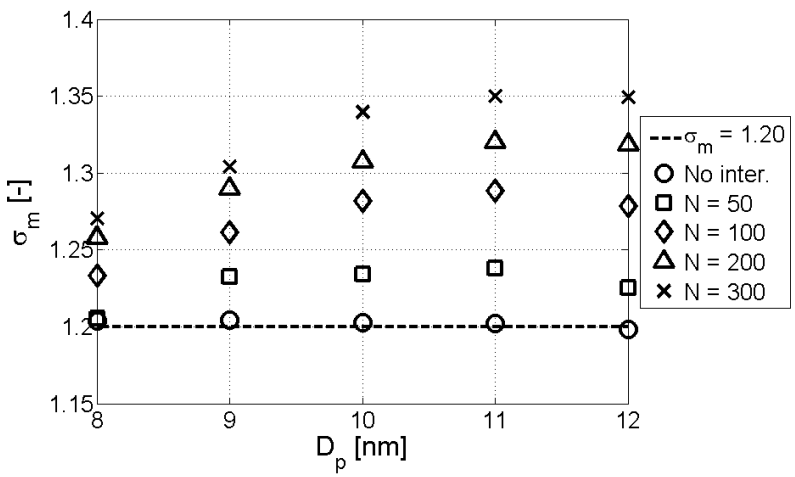

FIGURE 7. (a) $D_{m}$ versus $D_{p}$ and (b) $\sigma_{m}$ versus $D_{p}$, as a function of the number of MNCs (cluster type II) when including both the magnetic anisotropy $\left(K=3 \times 10^{4} \mathrm{~J} / \mathrm{m}^{3}\right)$ and dipole-dipole interactions: (open squares) $N=50$, (open diamonds) $N=100$, (open triangles) $N=200$, (crosses) $N=300$. The open circles show the simulated values for the noninteracting MNCs with no anisotropy. The dashed lines represents $D_{m}=D_{p}$ and $\sigma_{\mathrm{m}}=\sigma_{\mathrm{p}}$.

Since the standard deviation $\sigma_{p}$ can vary with the nanoparticle preparation method, the effect of this parameter is illustrated in Figs. 8 and 9 for clusters of type I and II, respectively. Similarly to what was observed in Figs. 6 and 7 for $\sigma_{p}=1.20$, other initial values of the physical standard deviation $\left(\sigma_{p}=1.05,1.10\right.$ and 1.15$)$ also result in an under- and overestimate of $D_{p}$ and $\sigma_{p}$, respectively, for both types of clusters.

A comparison of Figs. 8 and 9 shows that, for a given number of MNCs $(N=300)$ and anisotropy constant $\left(K=3 \times 10^{4} \mathrm{~J} / \mathrm{m}^{3}\right)$, a particle morphology of type II (Fig. 1 (b)) results in a larger error on the estimate of $D_{p}$ and $\sigma_{p}$ compared to a particle morphology of type I (Fig. 1 (a)). In both cases, the error on the estimate of $D_{p}$ slightly increases with $\sigma_{p}$. Interestingly, as the physical median diameter $D_{p}$ increases, the estimated parameters $D_{m}$ and $\sigma_{m}$ seem to respectively converge to similar values independently of the physical standard deviation $\sigma_{p}$. A similar behavior (not shown here) is also observed for smaller cluster sizes $(N=50,100,200)$. 
(a)

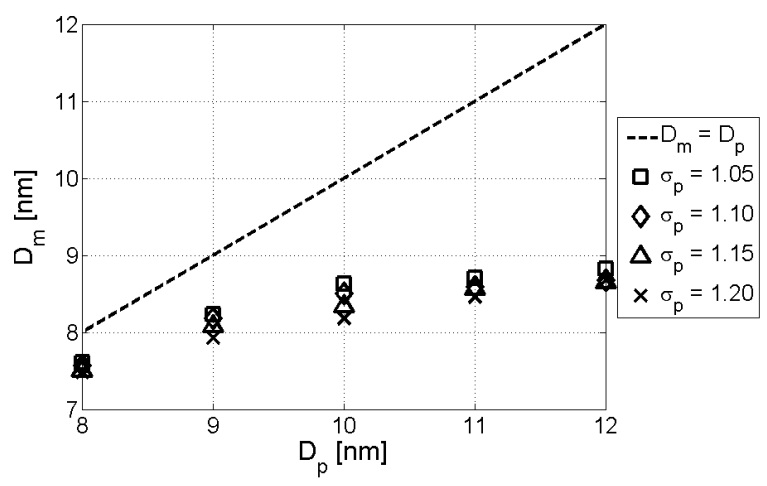

(b)

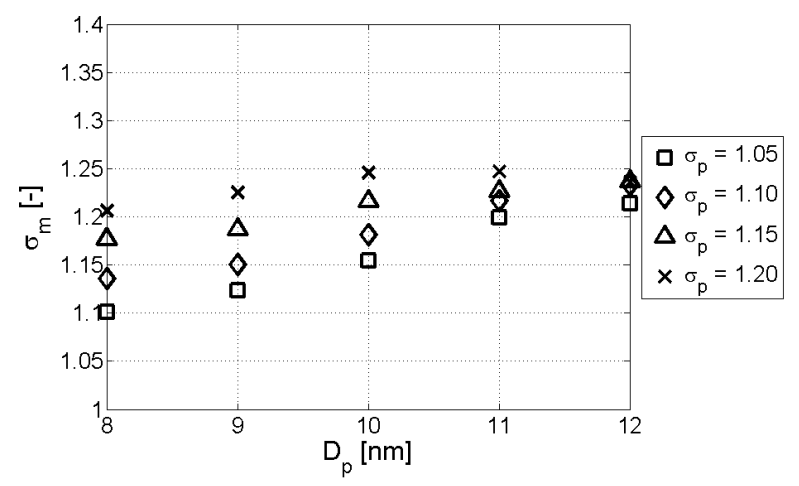

FIGURE 8. (a) $D_{m}$ versus $D_{p}$ and (b) $\sigma_{m}$ versus $D_{p}$, as a function of $\sigma_{p}$ (cluster type I with $N=300$ ) when including both the magnetic anisotropy $\left(K=3 \times 10^{4} \mathrm{~J} / \mathrm{m}^{3}\right)$ and dipole-dipole interactions: (open squares) $\sigma_{p}=1.05$, (open diamonds) $\sigma_{p}=1.10$, (open triangles) $\sigma_{p}=1.15$, (crosses) $\sigma_{p}=1.20$. The dashed line in (a) represents $D_{m}=D_{p}$.

(a)

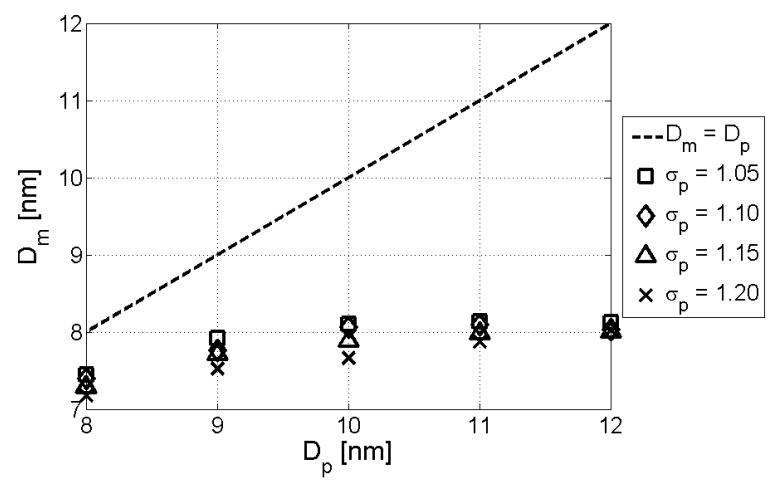

(b)

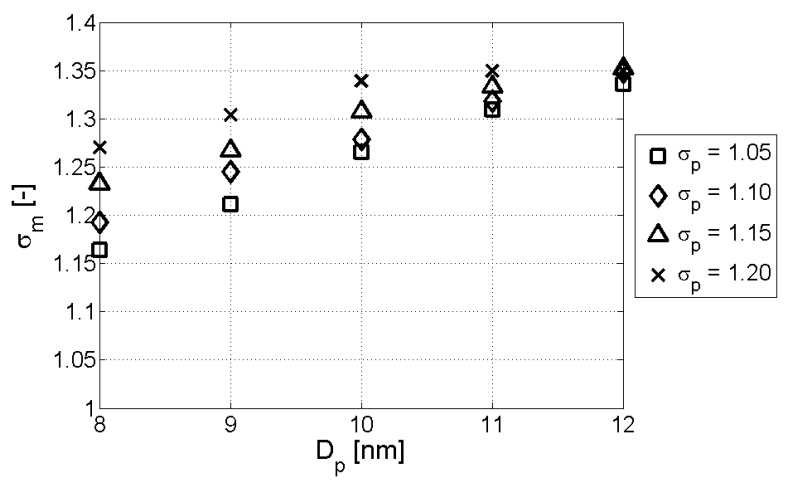

FIGURE 9. (a) $D_{m}$ versus $D_{p}$ and (b) $\sigma_{\mathrm{m}}$ versus $D_{p}$, as a function of $\sigma_{p}$ (cluster type II with $N=300$ ) when including both the magnetic anisotropy $\left(K=3 \times 10^{4} \mathrm{~J} / \mathrm{m}^{3}\right)$ and dipole-dipole interactions: (open squares) $\sigma_{p}=1.05$, (open diamonds) $\sigma_{p}=1.10$, (open triangles) $\sigma_{p}=1.15$, (crosses) $\sigma_{p}=1.20$. The dashed line in (a) represents $D_{m}=D_{p}$.

\section{CONCLUSIONS}

In this paper we have used a Monte Carlo simulation method to investigate the effect of uniaxial magnetic anisotropy and dipole-dipole interactions on the determination of the median diameter $D$ and geometric standard deviation $\sigma$ of magnetic nanocrystals (MNCs) contained in multicore particles. Two typical configurations of multicore particles are simulated: (I) random cluster of densely packed MNCs, and (II) MNCs deposited on the surface of a larger carrier sphere. The simulated magnetization data are then fitted with a Langevin equation weighted with a size distribution function, and the fitting parameters $D_{m}$ and $\sigma_{m}$ are compared to the real parameters $D_{p}$ and $\sigma_{p}$ used to generate the MNCs.

Our results show that fitting magnetization data with a Langevin model that neglects magnetic anisotropy and dipole-dipole interactions is not sufficient to obtain a proper estimate of the size distribution of the MNCs in multicore particles. We observe that both the magnetic anisotropy of the MNCs and the dipole-dipole interactions between MNCs modify the magnetization curve of multicore particles, which results in an erroneous estimate of the size distribution of the MNCs if these factors are not taken into account. When using only a Langevin model, the median diameter is constantly underestimated, whereas the geometric standard deviation is constantly overestimated for both particle configurations presented here. However, the extent of the error results from an interplay between particle morphology, number of MNCs contained in the particle, and magnetic properties of the MNCs. 
It is therefore relevant to extend the simulation method presented in this paper to other configurations and sizes of multicore particles. For instance, our preliminary results indicate that an error in the estimate of the size distribution of the MNCs even occurs for multicore particles containing less densely packed MNCs, e.g., where the MNCs are either separated by a thin (i.e., a few $\mathrm{nm}$ ) layer of oleic acid (e.g., Ref. 28) or homogeneously distributed in a carrier bead (e.g., Ref. 29). Smaller $(N=2-50)$ and larger $(\mathrm{N} \oslash 300)$ clusters are also being investigated. The results of this on-going work will be presented in details elsewhere.

The aim of this study is to provide a better understanding of the magnetization mechanisms in magnetic multicore particles, which may lead on one hand to a more reliable estimate of the size distribution parameters of the single-domain nanocrystals contained in the multicore particles, and on the other to practical guidelines in choosing multicore particle systems best suited to specific applications. This is expected to be particularly beneficial in applications such as biosensing, magnetic hyperthermia or magnetic imaging where it has been shown that a size distribution is often detrimental to the performances.

\section{ACKNOWLEDGMENTS}

Ångpanneföreningen's Foundation for Research and Development is gratefully acknowledged for financial support. The computations were performed on C3SE computing resources at Chalmers University of Technology.

\section{REFERENCES}

1. S. Sieben, C. Bergemann, A. Lübbe, B. Brockmann and D. Rescheleit, J. Magn. Magn. Mater. 225, 175-179 (2001).

2. N. L. Adolphi, D. L. Huber, J. E. Jaetao, H. C. Bryant, D. M. Lovato, D. L. Fegan, E. L. Venturini, T. C. Monson, T. E. Tessier, H. J. Hathaway, C. Bergemann, R. S. Larson and E. R. Flynn, J. Magn. Magn. Mater. 321, 1459-1464 (2009).

3. J.-F. Berret, N. Schonbeck, F. Gazeau, D. El Kharrat, O. Sandre, A. Vacher and M. Airiau, J. Am. Chem. Soc. 128, $1755-1761$ (2006).

4. M. Kettering, J. Winter, M. Zeisberger, S. Bremer-Streck, H. Oehring, C. Bergemann, C. Alexiou, R. Hergt, K. J. Halbhuber, W. A. Kaiser and I. Hilger, Nanotechnology 18, 175101 (2007).

5. S. Dutz, J. H. Clement, D. Eberbeck, T. Gelbrich, R. Hergt, R. Müller, J. Wotschadlo and M. Zeisberger, J. Magn. Magn. Mater. 321, 1501-1504 (2009).

6. M. Kallumadil, M. Tada, T. Nakagawa, M. Abe, P. Southern and Q. A. Pankhurst, J. Magn. Magn. Mater. 321, 1509-1513 (2009).

7. Y. R. Chemla, H. L. Grossman, Y. Poon, R. McDermott, R. Stevens, M. D. Alper and J. Clarke, Proc. Natl. Acad. Sci. USA 97, 14268-14272 (2000).

8. A. Prieto Astalan, F. Ahrentorp, C. Johansson, K. Larsson and A. Krozer, Biosens. Bioelectron. 19, $945-951$ (2004).

9. E. Heim, F. Ludwig and M. Schilling, J. Magn. Magn. Mater. 321, 1628-1631 (2009).

10. D. Eberbeck, F. Wiekhorst, U. Steinhoff, K. O. Schwarz, A. Kummrow, M. Kammel, J. Neukammer and L. Trahms, J. Magn. Magn. Mater. 321, 1617-1620 (2009).

11. R.E. Rosensweig, J. Magn. Magn. Mater. 252, 370-374 (2002).

12. R. M. Ferguson, K. R. Minard and K. M. Krishnan, J. Magn. Magn. Mater. 321, 1548-1551 (2009).

13. R. W. Chantrell, J. Popplewell and S. W. Charles, IEEE Trans. Magn. MAG-14, 975-977 (1978).

14. A. Ditsch, P. E. Laibinis, D. I. C. Wang and T. A. Hatton, Langmuir 21, 6006-6018 (2005).

15. R. W. Chantrell and E. P. Wohlfarth, J. Magn. Magn. Mater. 40, 1-11 (1983).

16. R. W. Chantrell, N. Walmsley, J. Gore and M. Maylin, Phys. Rev. B 63, 024410 (2000).

17. J. L. Dormann, F. D'Orazio, F. Lucari, E. Tronc, P. Prené, J. P. Jolivet, D. Fiorani, R. Cherkaoui and M. Noguès, Phys. Rev. B 53, 14291-14297 (1996).

18. J. Garcia-Otero, M. Porto, J. Rivas and A. Bunde, Phys. Rev. Lett. 84, 167-170 (2000).

19. M. Hanson, C. Johansson and S. Morup, J. Phys.: Condens. Matter 5, 725-732 (1993).

20. J.-O. Andersson, C. Djurberg, T. Jonsson, P. Svedlindh and P. Nordblad, Phys. Rev. B 56, 13983-13988 (1997)

21. C. G. Granqvist and R. A. Buhrman, J. Appl. Phys. 47, 2200-2219 (1976).

22. E. M. Claesson and A. P. Philipse, Langmuir 21, $9412-9419$ (2005).

23. V. Schaller, G. Wahnström, A. Sanz-Velasco, P. Enoksson and C. Johansson, J. Magn. Magn. Mater. 321, 1400-1403 (2009).

24. V. Schaller, G. Wahnström, A. Sanz-Velasco, S. Gustafsson, E. Olsson, P. Enoksson and C. Johansson, Phys. Rev. B 80, 092406 (2009).

25. D. P. Landau and K. Binder, A guide to Monte Carlo simulations in statistical physics, Cambridge University Press, Cambridge, 2000.

26. N. Metropolis, A. W. Rosenbluth, M. N. Rosenbluth and A. H. Teller, J. Chem. Phys. 21, 1087-1092 (1953).

27. M. Rasa, Eur. Phys. J. E 2, 265-275 (2000).

28. L. P. Ramirez and K. Landfester, Macromol. Chem. Phys . 204, 22-31 (2003).

29. G. Fonnum, C. Johansson, A. Molteberg, S. Morup and E. Aksnes, J. Magn. Magn. Mater. 293, 41-47 (2005). 